\title{
P66 Mathematical Model of the Renal Microcirculation and Effects of Chronic Kidney Disease
}

\author{
${ }^{1}$ King's College London \\ ${ }^{2}$ Department of Bioengineering, Imperial College London \\ ${ }^{3}$ Department of Vascular Biology and Inflammation, King's College London
}

Nikolaos Fountoulakis ${ }^{1, *}$, Karla Sanchez-Cazares ${ }^{2}$, Kim Parker$^{2}$, Janaka Karalliedde

\section{ABSTRACT}

Background: Diabetes is one of the leading causes of chronic kidney disease (CKD) worldwide. CKD is directly linked to increased morbidity and mortality. The role of vascular changes in diabetes and underlying mechanism of kidney disease is not well understood.

Methods: We present a mathematical model of the small arteries in the kidney incorporating anatomical and dynamic features. It consists of a symmetrical bifurcating treeself-similar in length, cross-sectional area and wave speed. Each generation is related to the previous one by the scaling factors e.g. $\ln +1=\lambda \ln$, this gives properties (e.g. surface area, resistance) for each generation by a priori knowledge of the renal artery. We assume the flow is one-dimensional and laminar. Vessel walls are treated as porousmedia to find the glomerulus transmural flux. Effects of compliance are introduced by a pressure-area relationship based on circumferential stress in thin vessel walls. The set of ordinary differential equations is solved numerically.

Results: The results are in accordance with physiological measurements and indicate that pressure in the vasculature is highly sensitive to changes in vessel geometry, which also affects the transmural flux in the glomerulus. Changes in the structure of the arterial wall (e.g. Young's modulus) alter the dynamics of the flow with an increased effect in the micro-circulation.

Conclusion: This is a functional model describing the behaviour of the small vasculature in the kidney. The model is computationally inexpensive, can be used for analysis and tested with in vivo data in pathological conditions.

(C) 2019 Association for Research into Arterial Structure and Physiology. Publishing services by Atlantis Press International B.V.

This is an open access article distributed under the CC BY-NC 4.0 license (http://creativecommons.org/licenses/by-nc/4.0/) 